\title{
Capítulo 7 Marcos analíticos de Las teorías de PAZ
}

Hay dos autores que son de necesaria referencia a la hora de tratar la sistematización de las teorías sobre la paz: Johan Galtung y Vincenç Fisas Armengol. Al primero se le reconoce el aporte de la distinción entre modelos de paz negativa y paz positiva, de los que se han derivado toda una serie de desarrollos esquemáticos para la clasificación de procesos e iniciativas de paz. De mayor fondo es el trabajo en el que realiza una de las primeras clasificaciones teóricas de lo que se venía tratando en la irenología ${ }^{6}$, que sirvió de base a la Unesco para definir políticas para el trabajo en esta área, con especial acento en la distinción entre la violencia directa y la estructural. Este elemento permitió transitar de la mirada clásica de la guerra, hacia la del conflicto y así poder soportar toda una serie de propuestas de investigación que buscan acercarse más a los elementos de carácter estructural que generan la violencia, al igual que plantear una gama amplia de estrategias para abordar este fenómeno más allá del enfrentamiento.

Por su lado, Vincenç Fisas realiza un trabajo en torno a la Escuela de Cultura de Paz de la Universidad de Barcelona, que ha servido de referente a quienes hoy quieren trabajar los temas de paz y conflicto, para lograr recoger sistemáticamente, y en una versión actualizada, las tendencias en la investigación y propuestas en torno a los procesos sociales que retoman estos dos conceptos. Su juicioso estudio de los años ochenta: Introducción al estudio de la paz y de los conflictos, es el

6 Referencia al tratado o conocimiento sobre los elementos que permiten a los grupos sociales obtener la paz, haciendo referencia a la acepción griega que significa 'paz': eirene. 
que servirá de referencia especial en este trabajo, principalmente por el análisis sistemático de lo que ha sido la investigación sobre la paz y, más aún, por el modo en que se concibe en relación con diferentes corrientes de pensamiento que le sirven de correlato. Este autor logra recoger en un solo texto relaciones de la mirada interdisciplinaria en el tratamiento de los conflictos, con especializaciones como las de las relaciones internacionales, la polemología, el estudio de los conflictos, los estudios estratégicos y la irenología; todos ellos, abrevando de posturas epistemológicas disímiles, desde el estructuralismo al conductismo, o desde la teoría de sistemas al posbehaviorismo, pasando por muchas otras.

Con estos referentes puede darse cuenta de un tipo particular de lectura de los procesos sociales latinoamericanos, en el marco del conflicto social del periodo de estudio, en especial si se tiene en cuenta que, por ejemplo, Galtung fue partícipe de la discusión con los teóricos de la dependencia, momento en el que fue reconocido por sus aportes metodológicos en el abordaje de la medición del desarrollo como proceso social de carácter diacrónico. Con ello se quería lograr un mayor acercamiento a lo que debía ser una ciencia social nomotética que facilitara el desarrollo de propuestas de cambio, minimizando los impactos de este. En ese escenario de lo metodológico se encontraba con pensadores de la talla de Peter Heintz, Óscar Cornblit, Torcuato di Tella, Ezequiel Gallo y Manuel Mora y Araújo, que tenían su correlato, en lo teórico, a Fernando Henrique Cardoso, Enzo Faletto, Torcuato di Tella, José Luis Reyna y Glaucio Ary Dillón Soares?

Por tanto, ambos autores y sus textos cumplen con dos elementos importantes de la metodología de esta tesis: incidir tanto en las formas de pensar como de actuar en el contexto latinoamericano en el periodo de estudio, y ser representativos de una línea de pensamiento, con un carácter sistemático en su trabajo y aplicación; es decir, han servido de marco interpretativo del conflicto en Latinoamérica y han sido referentes tanto de Estados como de grupos y movimientos sociales a la hora de actuar frente al conflicto en nuestro territorio.

Así las cosas, y para los propósitos del análisis propuesto en el trabajo, a continuación se presentan los referentes sobre los cuales se funda el análisis del conflicto que modela los discursos de paz, que más adelante servirán para mostrar

7 Veánse las memorias del Encuentro sobre Sociología del Desarrollo del Centro Latinoamericano de Investigaciones en Ciencias Sociales y la Unesco, publicadas en Buenos Aires en 1970 y realizado en Río de Janeiro en 1968. 
algunas consecuencias epistemológicas en torno a la función social que han cumplido los diferentes tipos de discurso analizados en la configuración actual de nuestra realidad, en especial en ese marco de abordaje de los conflictos.

\section{Periodización de LA INVESTIgación Y discursos}

\section{SOBRE LA PAZ Y EL CONFLICTO}

Retomando a Fisas, quien deriva su periodización de todo un contexto de debate en torno a los estudios sobre la paz y los conflictos y que, además, pasa por los diferentes encuentros mundiales y regionales en el tema y por la influencia de los organismos internacionales en su posicionamiento y legitimación, pueden plantearse cuatro grandes periodos, tomados cada uno en torno a una década, que sirven de marco evolutivo para el análisis del conflicto y de la paz.

El primero de ellos responde a la posguerra, es decir, comprende los años cincuenta, cuando como modelo de gestión de los conflictos se establece un sistema internacional que permita ejercer control sobre los Estados y que se centra en la concepción de la violencia como violencia física y directa. Allí la literatura hegemónica está relacionada con los procesos de rearme y desarme.

El segundo periodo sobre el que se construyen los paradigmas de este campo que expresa la tensión entre paz y conflicto es el de los años sesenta, cuando emerge claramente el concepto de violencia estructural, que tiene mucha relación con el eje de esta tesis, dado que el fondo del análisis se encuentra en el peso que tiene el debate en torno al desarrollo y el subdesarrollo, del cual deriva como modelo de paz el abordar las necesidades humanas como fundamento del cambio social, en un marco necesario que es el de la justicia social.

En los años setenta se hace fuerte la concepción de la necesidad de un orden mundial, en el que la guerra fría es parámetro en los procesos de gestión sociopolítica de los conflictos; sin embargo, a la vez se introducen metodologías de trabajo y se construyen abundantes teorías sobre cómo es posible el diseño de futuros alternativos que reconfiguren los problemas de las sociedades, y de esa manera se conminen las amenazas a la paz en el nivel interno a los Estados; es decir, los modelos estables de sociedad son la discusión sobre la cual se soportan las estrategias de acción política de los Estados, claro está que bastante polarizados por el contexto mismo de la guerra fría. 
Finalmente, en la periodización que se deriva del trabajo de Fisas, los años ochenta están caracterizados por el trabajo en escenarios sociales, políticos, educativos y comunicativos de organizaciones sociales que propenden a la educación para la paz, como modelo alternativo y a la vez estructural para generar los cambios que se requieren en las sociedades. Esto tiene como fin transformar el conflicto violento en un tipo de conflicto constructivo, en cuanto se comprende el valor de la diferencia, la tolerancia y el reconocimiento del otro como sujeto de derechos.

Llama la atención la asociación entre las formas en que la violencia se expresa para cada periodo y cómo las teorías de la paz encuentran propuestas de superación del esquema que no dejan de sugerir cómo dichos modelos surgen de un proceso evolutivo del pensamiento sobre la paz y los conflictos que se derivan de la maduración de las sociedades para abordarlo. En ese sentido, cada periodo incorpora del anterior la superación del modelo de conflicto subyacente, abriendo así ventanas a nuevos escenarios para ser abordados en la gestión de dichos conflictos. Claro está que también es posible realizar una lectura frente a núcleos de preocupación en el debate público, que Fisas relaciona con suficiente profundidad respecto de las organizaciones y los temas de investigación en torno a la paz.

Es claro que con esta periodización no se plantea la no existencia de modelos y desarrollos anteriores o posteriores en los temas aquí tratados, pero sí se resalta que son retomados en la medida en que se realiza un análisis crítico de estos, en especial sobre la producción sobre la polemología tanto clásica y conservadora, aquella que incorporando la conciencia de que el fenómeno de la guerra nos puede llevar a la "paz perpetua", en términos de Kant, derivó en desarrollos acerca de la gestión de los tratados de paz y estructuras políticas que disminuyeran los riesgos de la guerra como modelo de acceso y control del poder.

\section{Caracterización de los marcos de interpretación Y ANÁLISIS DE LOS CONFLICTOS}

Como se ha venido mostrando, toda investigación y propuesta de modelo de paz tiene de suyo una concepción de conflicto que abreva de escuelas de pensamiento, pero que al mismo tiempo aporta nuevos significantes y significados a estas respecto del análisis de la sociedad y los modelos de acción derivados. 
El primer punto por resaltar sobre el análisis de las investigaciones sobre la paz es su carácter interdisciplinario, del cual muchas veces en las propuestas se diluye el énfasis disciplinar para dar lugar a construcciones que van más allá de visiones reduccionistas acerca de un modelo teórico o conceptual, muchas de ellas derivadas de la puesta en práctica y sistematización de experiencias de trabajo en las que la participación de múltiples actores, de carácter político, académico, social y económico, hacen de estos nuevos marcos de análisis muchas veces propuestas que se acercan a conocimiento transdisciplinario.

En ese sentido, en la figura 2 se representan diferentes fuentes disciplinares y sus temas en torno a la investigación sobre la paz.

Figura 2. Aportes interdisciplinares en la construcción de los marcos de interpretación y análisis de los conflictos

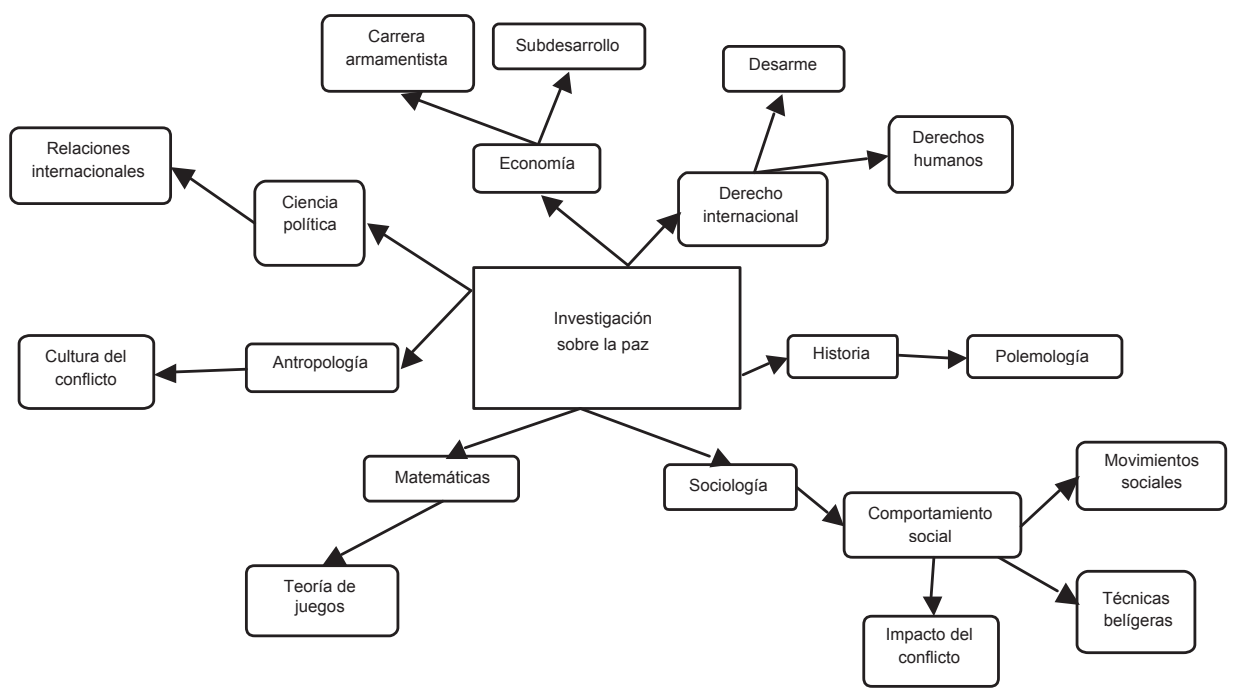

Fuente: autor, con base en el texto de Fisas (1987): Introducción al estudio de la paz y los conflictos.

Sin ser enumerativo de la totalidad de aportes que se han dado, desde el orden disciplinar se resalta el aporte clásico de lo que hoy denominamos la ciencia politica y el derecho internacional, de las cuales la filosofía ha sido interlocutora permanente, en especial en el enfoque nomotético, y en cuyo contexto las temáticas 
de las relaciones internacionales y los derechos humanos son preponderantes a la hora de abordar el tema de los conflictos. Incluso, dicha reflexión ha permitido ampliar el espectro de acción sobre la vida social, sobre todo en lo concerniente a los derechos de primera a cuarta generación, en los que la misma configuración de los Estados ha tenido que reconsiderarse para abordarlos, por lo cual la relación entre ellos adquiere nuevos sentidos. En este último escenario, lo que en un principio monopolizó el trabajo en torno al derecho internacional, el desarme, fue base para que se generaran estructuras para actuar sobre otros temas como la cultura, la educación, el desarrollo, el medio ambiente, etc., los cuales sirvieron, a su vez, de escenarios para la acción en territorios concretos que incidían en términos de las relaciones de fuerza puestas en juego en la resolución de los conflictos.

Se reconoce el significativo aporte de la economía, en cuanto disciplina que posicionó los debates en relación con el subdesarrollo en el mundo, donde el análisis crítico frente a las consecuencias de la carrera armamentista abre la posibilidad de relativizar su importancia y, así, sustentar estrategias paralelas, rentables económica y humanamente en la resolución de los conflictos. Esto derivó hasta en el surgimiento de premios de economía alternativos a los modelos que se planteaban como insuficientes a la hora de resolver la problemática del mundo. El énfasis que la economía y el derecho tuvieron en la modelación de los procesos gubernamentales permitió que los desarrollos de estas disciplinas incidieran fuertemente tanto a favor como en contra de los modelos de cambio visualizados socialmente como necesarios. Por su carácter estratégico, terminaron realizando un aporte en doble vía: una primera vía desde el análisis económico de los fenómenos de la vida política y social, sustentando modelos de desarrollo y crecimiento adecuados a las nuevas lógicas del orden mundial, a la vez que soportando teorías alternativas de organización socioeconómicas que atendieran los fenómenos de hambre y pobreza disonantes a la hora de evaluar la efectividad de los primeros; una segunda vía, la de incorporar los aportes de otras disciplinas, en especial las de las otras ciencias sociales, incidiendo en la estructuración de modelos complejos de evaluación del impacto de las medidas gubernamentales.

En este contexto surge el aporte sobre la importancia de modificar la concepción de necesidad e incorporar la adjetivación de humana que transformó visiblemente el análisis de la vida económica de los pueblos. Así las cosas, se introdujeron indicadores sobre el impacto en el ambiente y la ecología, así como sobre la seguridad y la participación social, entre muchos otros que hoy hacen parte 
de la evaluación del desarrollo. Se introducen así, de forma estructural, disciplinas como la geografía, la biología y la ecología, pues también las implicaciones de sus saberes modificaban las tesis de la economía clásica, liberal y totalizante de la vida social.

El aporte de la sociología a las teorías de la paz lo centra Fisas en el análisis del comportamiento social. En ese sentido retoma ciertas escuelas de la disciplina que trabajan articuladamente con teorías de la psicología y la antropología. Algunos de los temas de mayor frecuencia en el análisis se relacionaban con el impacto del conflicto en la vida social, donde caracterizaciones como las de $\mathrm{Co}^{-}$ ser y Dahrendorf fueron clásicas, dado que introdujeron nuevos enfoques frente a cómo se concebía el conflicto, sobre todo en su función integradora de los grupos sociales, poniendo escenarios de análisis para el conflicto social más allá de la ya macartizada lucha de clases, pero sin dejar de lado su papel preponderante en el cambio social. De allí que el tema de los movimientos sociales tuviese tanta relevancia en los análisis posteriores del papel de la sociedad civil en la transformación de la realidad social.

La caracterización de las "técnicas belígenas" fue un eje al que se le encargó a sociólogos el trabajo investigativo, que con la antropología y el concepto de cultura, y la psicología con los patrones de conducta individual que hacen presencia en la vida social, fueron dando lugar a los modelos de análisis sobre la cultura del conflicto, que tienen como propósito intentar dar respuesta al imaginario social del carácter conflictivo del individuo y, por tanto, el carácter de necesidad del conflicto y la violencia en la vida social.

No son despreciables los aportes de las matemáticas mediante los planteamientos sobre la teoría de juegos, que más modernamente ha evidenciado usos en la gestión de los conflictos sociales, ambientales, económicos, etcétera. Asimismo, las escuelas analizadas por los teóricos de la paz identifican el aporte de la historia en la polemología, si bien para otras escuelas los mismos métodos críticos de la historia lograron abordar otros escenarios explicativos de los procesos sociales ajenos a la historiografía de las guerras en la historia.

Al respecto, Galtung resalta de este proceso de aporte interdisciplinar a la investigación sobre la paz los diferentes esfuerzos de investigación orientada hacia los problemas, que pone de presente un nuevo paradigma, donde su mismo estatus epistemológico de legitimidad y validez se modifica, rompiendo con la estricta lógica de una ciencia que dirige la acción hacia un pensamiento que 
incorpora conocimientos adquiridos por distintas vías y de forma dialéctica asume sentidos que se van actualizando en la experiencia.

Sin embargo, esta afirmación contrasta con la sistematización de los aportes extraídos de dicho trabajo investigativo - pues acogen selectivamente sus productos y derivan, finalmente, contribuciones desde el orden nomotético, a partir del cual se busca dirigir la acción-, frente a aportes más complejos que incluso en relación con el desarrollo le dan un papel a la construcción social preponderante en la estructuración de la vida. Fisas (1987) analiza frente a la relación entre paz y desarrollo lo siguiente:

Por eso, dentro de los estudios que se llevan a cabo sobre los temas del desarrollo, la investigación sobre la paz conecta mejor con la corriente de pensamiento normativa que recalca la importancia de las necesidades humanas básicas, un medio ecológico sano y la autoconfianza. Esta concepción del desarrollo contrasta enormemente con el paradigma convencional de la "modernización y el crecimiento" (p. 78).

En ese sentido, se resume que la investigación sobre la paz puede ser catalogada como conceptual, normativa y empírica. Con Marek Thee, Fisas (1989) la define elocuentemente como:

- Humana en sus objetivos

- Cientifica en sus métodos

- Pragmática en sus experimentaciones

- Internacional por naturaleza

- Global en perspectiva

- Orientada hacia la acción en cuanto a su aspiración (p. 18).

\section{EN TORNO AL CONCEPTO DE VIOLENCIA ESTRUCTURAL}

En un texto clásico intitulado Contribución especifica de la irenología al estudio de la violencia: tipologías (Unesco, 1981, pp. 92-106), Galtung hace la distinción conceptual de tipos de violencia, introduciendo una gama de posibilidades esquemáticas para su clasificación, cuyo fondo se basa en un enfoque negativo y otro positivo desde el cual la clasificación asume un carácter político en la 
perspectiva de proponer a los seres humanos la posibilidad o no de evitarla o de propiciar la realización humana.

En torno al enfoque negativo, desarrolla la tipología que da cuenta desde la violencia clásica hasta la estructural. Los principios clasificatorios dicotómicos son: por un lado, en la medida que la violencia se concibe como acción o no; por otro, si se concibe como un acontecimiento o algo permanente, como se muestra en la siguiente tabla.

Tabla 1. Primera tipología

\begin{tabular}{|c|c|c|}
\cline { 2 - 3 } \multicolumn{1}{c|}{} & $\begin{array}{c}\text { Violencia como } \\
\text { acontecimiento }\end{array}$ & $\begin{array}{c}\text { Violencia como } \\
\text { algo permanente }\end{array}$ \\
\hline Violencia como acción & Tipo I & Tipo II \\
\hline Violencia como no-acción & Tipo III & Tipo IV \\
\hline
\end{tabular}

Fuente: Galtung (1981, p. 94).

Según esta propuesta de clasificación, el tipo I sería la violencia clásica (que denominará directa), y el IV la violencia estructural. Respecto al tipo IV, señala:

Sería el caso puro de violencia estructural, ya que si no hay autor, sino un estado permanente de violencia que no puede calificarse de natural (en el sentido de ser "inevitable"), la violencia debe ser algo inherente a la estructura social (Unesco, 1981, p. 94).

Llama la atención que para poder distinguir la violencia de la necesaria existencia de un agresor era significativo poder analizar la problemática. La idea de encontrar un culpable y analizar las lógicas que lo llevaban a ejercer la violencia copaba gran parte de la investigación, para poder prevenir la violencia, las guerras o los conflictos; sin embargo, era claro que esta visión era insuficiente para poder comprender realmente lo que ocurría al observar la complejidad de la dinámica social.

Por tal razón, Galtung introduce el enfoque positivo, que en última instancia es la propuesta de cómo abordar la violencia y el conflicto como escenario constructivo, por la vía de concebir la violencia como algo evitable. En este contexto se generan las condiciones sociales necesarias para que no sea apetecible ningún tipo de violencia, a lo que habría que agregar que dichas condiciones 
darían lugar a una maduración de las formas de afrontar el conflicto, dándole salidas sociales creativas para que no genere tipos de violencia, en especial directa y, más allá, estructural. Es ese contexto, Galtung (1981) dice:

Avancemos ahora tomando como punto de partida la idea de violencia como algo evitable que obstaculiza la realización humana. Entenderemos por autorrealización humana la satisfacción de las necesidades del hombre, y utilizaremos la siguiente lista de necesidades básicas, materiales y no materiales (p. 96).

Entre ellas, las de supervivencia, fisiológicas, ecológicas, sociales, de libertad, políticas, jurídicas, de trabajo y de relación con la sociedad, con otros, con la naturaleza y consigo mismo. Esta perspectiva y estructura de clasificación introdujo varios trabajos que buscaban darle mayor profundidad, tal como el trabajo del chileno Manfred Max Neef, que con el apoyo de Antonio Elizalde y Martín Hopenhayn dieron lugar a una obra de alto impacto en los temas del desarrollo en Latinoamérica para los años ochenta, como fue Desarrollo a escala humana, cuyo objetivo era el siguiente:

Integrar líneas de reflexión, de investigación y de acción que puedan constituir un aporte sustancial para la construcción de un nuevo paradigma del desarrollo, menos mecanicista y más humano [...] El texto resultante aspira a tener como interlocutores a agentes del desarrollo regional, planificadores y políticos, grupos de desarrollo local, académicos de diferentes disciplinas relevantes para el desarrollo, foros internacionales y profesionales e intelectuales dedicados a pensar caminos de humanización para un mundo en crisis (Max Neef, 1993, p. 18).

Como se puede ver, esta apropiación de la concepción positiva para enfrentar la violencia estructural se convirtió rápidamente en una propuesta de desarrollo alternativo, de la que se fundamentaron varias escuelas académicas en trabajo sobre desarrollo. Es claro que el mismo planteamiento también tiene su origen en el mismo contexto latinoamericano, si se observa que el debate del que participa Galtung venía desde los años setenta en este continente; es decir, estas ideas se pueden concebir como un momento de maduración en dos contextos geográficos diferentes, de un principio de análisis común, que para los propósitos de este 
trabajo permite mostrar cómo desarrollo y conflicto son correlatos de un mismo campo de conocimiento.

En la tipologización de Galtung en torno a la violencia estructural se despliega una matriz de análisis de otros cuatro tipos de violencia desde este enfoque positivo, que son el de violencia clásica, el de pobreza como privación de necesidades materiales básicas, el de represión como privación de derechos humanos y el de alienación como privación de necesidades superiores. Con ello pone el dedo en la llaga sobre la responsabilidad social de los gobiernos como agentes de cambio para construir escenarios que permitan condiciones de desarrollo humano en un espectro mucho más grande que el de la sobrevivencia. De allí abrevarán diferentes actores sociales en términos de la justificación de su accionar, incluso en el mismo uso de las armas, como ocurrió con la conformación de guerrillas en Latinoamérica.

Cuando aborda el tema de la paz, muestra, por tanto, que se puede hacer una diferenciación de la paz negativa frente a la positiva, que pone de presente que la violencia de los movimientos insurgentes o de liberación es igualmente directa, producto de que una paz que profundiza la violencia estructural no es posible concebirla sino como una "parodia" de paz; es decir, esta última conduce nuevamente a la primera. Es de aclarar que aquí se muestra de forma sistemática lo que el contexto sociopolítico latinoamericano venía argumentando en su accionar, lo cual sirve para ver cómo mediante diversas vías la estructura lógica de concepción de la realidad conlleva conclusiones similares.

La conclusión de dicha propuesta gira en torno a que la violencia engendra violencia, lo que es clave a la hora de superar la idea de que el hombre es violento por naturaleza. Desde esta última condición, ahí sí que tendría que pensarse en el fin de la historia que muchos autores han intentado posicionar en la actualidad; sin embargo, establecer la problemática en el entorno sociopolítico abre las compuertas a procesos transformadores de construcción social, en los que el ser humano y no el azar es el protagonista principal de su propia realización.

\section{ESQUEMATIZACIÓN DE LAS TEORÍAS DE PAZ}

Siguiendo a Groff y Smoker (1996), puede plantearse cómo se han fijado esquemáticamente las clasificaciones de las teorías de la paz, las cuales se convierten en una matriz de análisis que ya sea en sus componentes o en sus 
interrelaciones viene siendo utilizada por gran parte de los teóricos y consultores en los procesos de paz, en diferentes sitios del mundo. Latinoamérica sigue siendo uno de los lugares privilegiados para la puesta en juego de procesos fundamentados en tales clasificaciones.

Por una parte, se propone la utilización de las nociones de paz positiva y paz negativa como marcos de clasificación más amplios de otros tipos teóricos que se valoran, según se veía con Galtung, aunque también se organizan bajo la premisa de su origen histórico como profundización de la comprensión de la problemática de la paz.

En ese sentido, en el marco de la paz negativa se organizan los modelos de ausencia de guerra y balance internacional de fuerzas, donde el modelo de las $\mathrm{Na}$ ciones Unidas es paradigmático para explicar todo un proceso de concientización de que la alternativa de la paz perpetua, como la denominó Kant, es un camino expedito para la desaparición de la especie humana, junto con otras que en el camino serían también destruidas.

En el de la paz positiva, se inicia con el manejo de la violencia estructural, la paz feminista, la paz holística Gaia (ambiental) y la paz holística interior y exterior. Allí se incorpora todo el trabajo que las organizaciones no gubernamentales han venido haciendo para posicionar elementos de reflexión para identificar nuevos escenarios de violencia y conflicto, como en el caso de la paz feminista, que más que poner la discusión en las diferencias de género, invita a prestar atención a todas las microestructuras de la violencia, evidentes en la cotidianidad, pero con significativos efectos a nivel estructural. De la misma forma, se ven los avances que en temas ambientales y de derechos se vienen trabajando, como se observa en expresiones significativas derivadas de la teoría de la complejidad con Edgar Morin, y que en el marco del tipo de discursos aquí descrito se puede retomar hoy en día con las ecologías de Leonardo Boff en Latinoamérica (ecología ambiental, social, mental e integral).

El ámbito de la vida interior del ser humano es otro escenario que se constituye en categoría de análisis y acción frente al tema de los conflictos y la paz. En el modelo que hemos venido presentando (y que se puede ver sintéticamente en la figura 3), este escenario aparece desde dos perspectivas que se integran y tocan transversalmente.

En ese sentido, todos los anteriores tipos de paz se interrelacionan desde otros dos marcos de clasificación: la paz externa e interna. En el primero se 
abordan los niveles de relacionamiento de los seres humanos, referentes al medio ambiental, el global, el internacional, las relaciones entre y en el interior de los Estados, las relaciones comunitarias familiares y personales. Finalmente, en el segundo marco se aborda la paz interior.

Figura 3. Conceptos en la evolución de la paz

\begin{tabular}{|c|c|c|c|c|c|c|c|}
\hline & $\begin{array}{l}\text { Ausencia } \\
\text { de guerra }\end{array}$ & $\begin{array}{l}\text { Balance } \\
\text { de fuerzas }\end{array}$ & $\begin{array}{c}\text { No } \\
\text { violencia } \\
\text { estructural }\end{array}$ & $\begin{array}{c}\mathrm{Paz} \\
\text { feminista }\end{array}$ & $\begin{array}{c}\text { Paz } \\
\text { holística } \\
\text { Gaia }\end{array}$ & $\begin{array}{c}\text { Paz holística } \\
\text { interior y } \\
\text { exterior }\end{array}$ \\
\hline \multirow{7}{*}{ 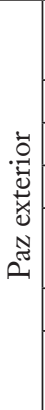 } & $\begin{array}{c}\text { Medio } \\
\text { ambiental }\end{array}$ & & & & & & \\
\hline & Global & & & & & & \\
\hline & Internacional & & & & & & \\
\hline & Entre los Estados & & & & & & \\
\hline & $\begin{array}{l}\text { En el interior } \\
\text { de los Estados }\end{array}$ & & & & & & \\
\hline & Comunitaria & & & & & & \\
\hline & $\begin{array}{c}\text { Familiar } \\
\text { e individual }\end{array}$ & & & & & & \\
\hline & Paz interior & & & & & & \\
\hline
\end{tabular}

Fuente: Groff y Smoker (1996).

Cada cruce daría para analizar marcos discursivos desde los cuales se aborda la reflexión sobre la paz y los conflictos; es decir, al menos cuarenta y ocho tipos de discurso, formas de acción y necesidades de cambio son posibles de ser clasificadas por este esquema, que al tenor dan la idea de poder incorporar el espectro completo de las relaciones conflictivas presentes en nuestras sociedades, así como las propuestas de paz derivadas de ellas.

La ganancia de este tipo de propuestas se ha visto especialmente en escenarios de educación y de movimientos sociales preocupados por los temas de la paz. Estos esquemas facilitan su rápida memorización y, por tanto, se incorporan en los discursos cotidianos. Así, sirven de modelos paradigmáticos de interpretación de la realidad e inciden en la acción social de formas diversas.

Sin embargo, es importante llamar la atención sobre que la aparente sistematicidad y el amplio espectro de análisis no son sinónimos de profundidad en la comprensión y de eficacia en sus resultados. Si bien se asumen estructuras 
fuertes de análisis como el marco de la violencia estructural, lo asumen como estructura normativa de clasificación, fenómeno que se evidencia en las diferentes escuelas que se han venido presentando a lo largo de este capítulo. Así las cosas, varias de las disciplinas, escuelas de pensamiento y autores requieren ser retomados a la hora de abordar el campo de análisis de la violencia, el conflicto y la paz, donde la reconstrucción histórica debe verse nuevamente en su complejidad y análisis crítico, y no solo en aquello que aporta a la interpretación del conflicto como situación. La sociología y las ciencias sociales en general deben retomarse como teorías complejas sobre la sociedad. Donde el conflicto es uno de sus componentes, la teoría política debe servir para pasar del análisis a la asunción de posturas claras sobre los fenómenos de la realidad que propicien escenarios de construcción del cambio social, sin ocultar la importancia de tener en cuenta el efecto que la categoría poder tiene en dicha construcción; es decir, cada uno de los escenarios aquí propuestos, en una visión del fenómeno latinoamericano, requerirá ser contrastados en el marco de tensión del campo de conocimiento que se viene trabajando. 